Int. J. Dev. Biol. 56: 75-82

doi: $10.1387 / \mathrm{ijdb} .113443 \mathrm{~cm}$

\title{
An insulin-like peptide regulates size and adult stem cells in planarians
}

\author{
CLAIRE M. MILLER ${ }^{1}$ and PHILLIP A. NEWMARK ${ }^{*, 2}$ \\ ${ }^{1}$ Neuroscience Program, Medical Scholars Program, and ${ }^{2}$ Howard Hughes Medical Institute, \\ Department of Cell and Developmental Biology, University of Illinois at Urbana-Champaign, IL, USA
}

\begin{abstract}
Animal growth depends on nutritional intake during development. In many animals, nutritional status is uncoupled from moderation of adult stature after adult size is achieved. However, some long-lived animals continue to regulate adult size and fertility in a nutrition-dependent manner. For example, the regenerating flatworm Schmidtea mediterranea becomes smaller, or degrows, during periods of starvation. These animals provide an opportunity to readily observe adult stem cell population dynamics in response to nutritional cues. We explored the role of insulin signaling in $\boldsymbol{S}$. mediterranea. We disrupted insulin signaling via RNA interference and showed that animals, despite eating, degrew similarly to starved animals. Utilizing in situ hybridization and immunofluorescence, we assessed cellular changes in proliferative populations including the planarian adult stem cell population (neoblasts) and the germline. Both impaired insulin signaling and nutritional deprivation correlated with decreased neoblast proliferation. Additionally, insulin signaling played a role in supporting spermatogenesis that was distinct from the effects of starvation. In sum, we have demonstrated that insulin signaling is responsible for regulation of adult animal size and tissue homeostasis in an organism with plastic adult size. Importantly, insulin signaling continued to affect stem cell and germline populations in a mature organism. Furthermore, we have shown that adult organisms can differentially regulate specific cell populations as a result of environmental challenges.
\end{abstract}

KEY WORDS: insulin, planarians, adult stem cell, germ cell, spermatogenesis

\section{Introduction}

Animal growth requires the coordination of nutrient resource availability with stem cell dynamics. The insulin/IGF family is a conserved regulator of metabolism and moderates overall animal growth and development (Chan and Steiner, 2000; De Meyts, 2004). Insulin-like peptide signaling continues to control germline stem cell dynamics in adulthood (LaFever and Drummond-Barbosa, 2005; McLeod et al., 2010; Michaelson et al., 2010; Ueishi et al., 2009). Given that insulin/IGF signaling plays a significant role in cancer (Maki, 2010; Samani et al., 2007), the regulatory effects of this pathway on adult stem cell dynamics merit further exploration.

Though the roles of insulin/IGF signaling during development appear to be conserved across species, the actual number of insulin-like peptides and the specific functions of those peptides are highly divergent, particularly amongst invertebrates (Chan and Steiner, 2000). Invertebrate insulin-like peptides are commonly expressed within the nervous system and regulate growth and reproduction (Wu and Brown, 2006). In mammals, insulin growth factors (IGFs) play a role in the somatotropic axis of the pituitary gland and are expressed within the brain as well as other tissues (Daftary and Gore, 2005). Disrupted neuroendocrine insulin/IGF signaling during development results in decreased adult size in Drosophila Melanogaster (Chen et al., 1996; Ikeya et al., 2002) and several strains of dwarf mice (Tatar et al., 2003). Although the role of insulin/IGF signaling during embryonic and post-embryonic growth is clear, once adult size is attained, the continuing cellular effects of the growth-regulating aspect of the pathway are poorly understood.

In both Caenorhabditis elegans and D. melanogaster, insulinlike signaling acts upon adult germline stem cells to regulate proliferation. In $C$. elegans, two of 40 known insulin-like peptides support the L3/L4 stage germline expansion by signaling through the single insulin receptor (DAF-2) (Michaelson et al., 2010). In D.

Abbreviations used in this paper: dsRNA, double-stranded RNA; IGF, insulin growth factor; ILP, insulin-like peptide; INR, insulin receptor; INSL, insulin-like factor; RNAi, RNA interference; Tral, Trailer-hitch.

\footnotetext{
*Address correspondence to: Phillip A. Newmark. 601 S Goodwin Ave., Chem. Life Sci. Lab., room B107, Urbana, IL, 61801, USA. Fax: +1-217.244.1648. e-mail: pnewmark@life.illinois.edu
} 
melanogaster, insulin-like signaling mediates adult gametogenesis (LaFever and Drummond-Barbosa, 2005; Ueishi etal., 2009) as well as the ovarian proliferative reponse to nutrition (Drummond-Barbosa and Spradling, 2001; Hsu and Drummond-Barbosa, 2009; LaFever and Drummond-Barbosa, 2005). Furthermore, increased insulinlike signaling can partially rescue decreased germline proliferation due to poor nutrition (McLeod et al., 2010). Together, these findings indicate that insulin signaling may play a conserved role in the regulation of adult fertility and germline stem cell populations, in part through communication of nutritional state. However, further studies in other adult stem cell populations, including those in a non-reproductive context, are warranted.

The flatworm Schmidtea mediterranea is renowned for its regenerative capacity, which is dependent on a population of totipotent adult stem cells called neoblasts (Baguñá et al., 1989; Brøndsted, 1969; Newmark and Sánchez Alvarado, 2000; Wagner et al., 2011). This model organism is also intriguing because its adult size is effectively dissociated from age; size depends on nutritional status, such that fed animals grow, while starved animals shrink yet maintain their proportionality (degrow) (Baguñá and Romero, 1981; Newmark and Sánchez Alvarado, 2002; Oviedo et al., 2003; Wenemoser and Reddien, 2010). There are two strains of $S$. mediterranea: a sexually reproducing, hermaphroditic strain and an asexual, fissioning strain. These strains can be used to assess gene function during gametogenesis as well as in a context devoid of reproductive system development. The experimental ease with which adult stem cell and germline population dynamics can be observed (Eisenhoffer et al., 2008; Guo et al., 2006; Reddien et al., 2005b; Wang et al., 2007; Wang et al., 2010) make $S$. mediterranea an asset to studies of developmental and regenerative biology.

In the sexual strain of $S$. mediterranea, sexual development and gonad maintenance also appear to depend on metabolic state rather than age, as animals post-embryonically develop reproductive organs only

C. elegans

\section{Ligand-binding domain 1}
H. sapiens
S. mansoni
s. mediterranea

Ligand-binding domain 2 RNA (rRNA) serves as a loading control.

once they achieve an approximate size (Newmark and Sánchez Alvarado, 2002). Should the animal degrow past a certain size due to lack of nutritional intake, then its gonads regress. Gonads redevelop when adequate nutrition is once again available (Morgan, 1902; Wang et al., 2007). Though several genes required for proper

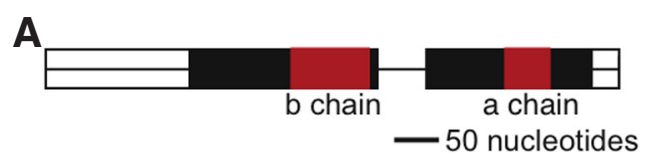

B
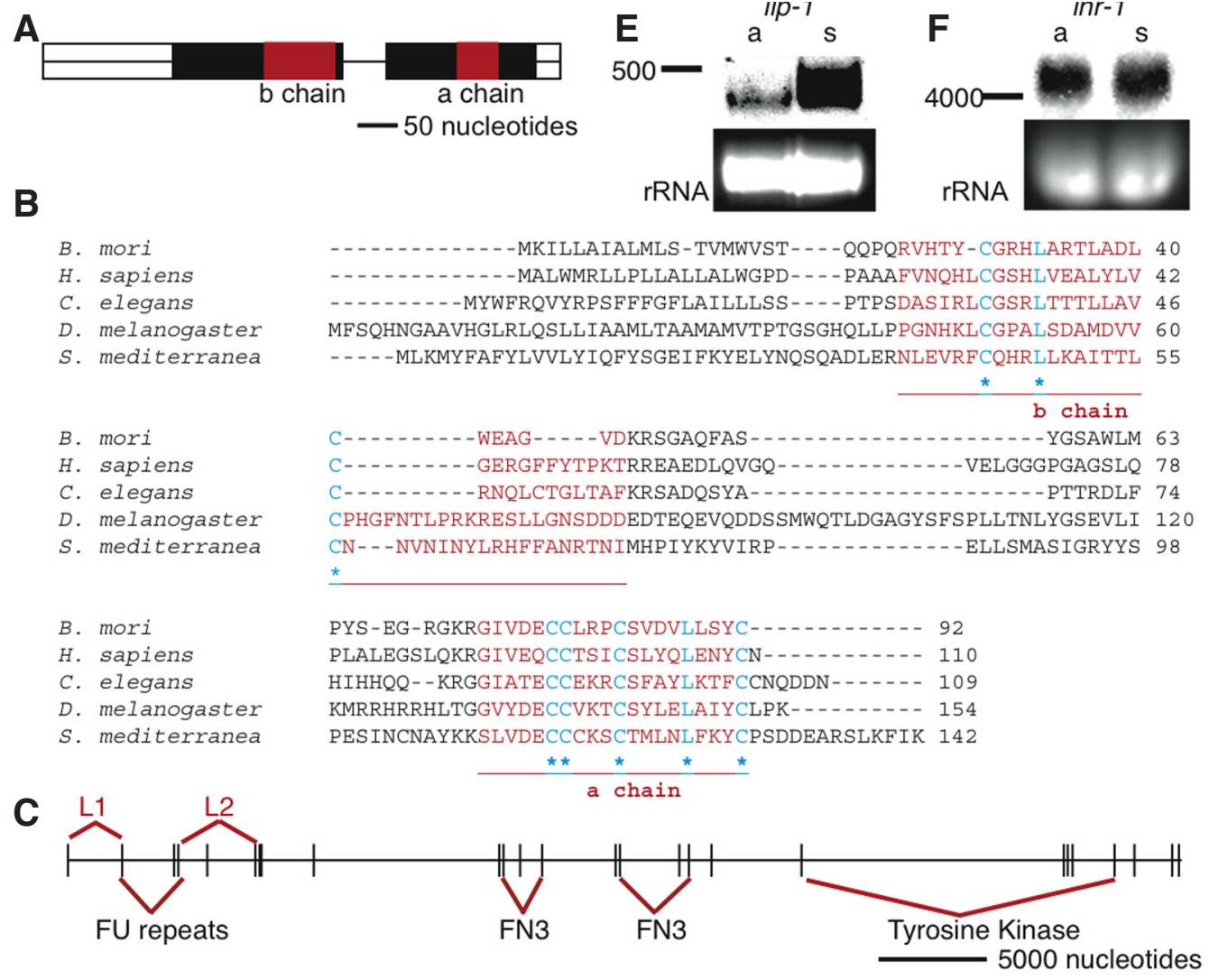

B. mori

H. sapiens

C. elegans

D. melanogaster

S. mediterranea

B. mori

H. sapiens

D. melanogaster

S. mediterranea
B. mori
H. sapiens
C. elegans
D. melanogaster
S. mediterranea

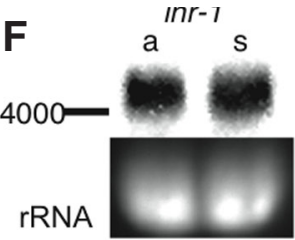
H. sapiens
NCSVIEGHLQILLMFKTRPEDFRDLSFPKLIMITDYLLLFRVYGLESLKDLFPNLTVIRG 60
S. mansoni -CTVIEG--DLFVVFTRIP - - -RDASLPFLREITGSLLVYDVEGPDDLSNLLPNLTLIRG 54
s. mediterranea -..-...-.--MVTLTS----EYSFPTLKEITGSLVIYYVKGTKTLGLYFPNLTIIRG 43

SRLFFNYALVIFEMVHLKE- - - - LGLYNLMNITRGSVRIEKNNELCYLATIDWSRIL- - 113 QTLVFGYAVVI - KSTSLKN - - - - IGLFSLRVIQQGGVRIDSNPQLCYVRTIDWDVIL - - 106 QTLAYNYAVVI KETNLLVINLFQYIGLVNLRLVMFGGVRIDNNPSLCYVNTINWTSIVRQ 103

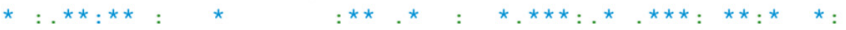
H. sapiens
S. mansoni
I INIRGGNNLAAELEA-NLGLIEEISGYLKIRRSYALVSLSFFRKLRLIRGETLEIG- - - 452
S. mediterranea LSIQEGOPEIVALLLDRAFANLRVIHHSLRIVRSSVLNNLNFLRHVRSIGOIGG- - - - - 453 ISIKEGGSSLSDILMK-AFSKLTIIENSLRIVKSSSITSLDFLRSLRI I KGNSANKNNNE 351
H. sapiens
S. mansoni
S. mediterranea

Fig. 1. Genes encoding an insulin-like peptide (ilp-1) and an insulin receptor (inr-1) are present in $S$. mediterranea. (A) Map of the ilp-1 gene sequence including untranslated regions (hollow boxes), an intron (line) and translated regions (shaded boxes) with the conserved $b$ and a chain sequences in red. (B) Alignment of selected insulin family member sequences with b and a chains indicated in red, and highly conserved residues, including six canonical cysteines, in blue. (C) Map of the inr-1 gene spanning 30,000 basepairs including: 26 exons (vertical lines) and 25 introns (horizontal lines); conserved domains marked in red. (D) Alignment of selected insulin receptor ligand-binding domains with conserved residues in blue and partially conserved residues in green. (E,F) Northern blots of ilp-1 and inr-1 probe on asexual (a) and sexual (s) planarians; ribosomal 
neoblast or germline function have been identified (Collins et al., 2010; Guo et al., 2006; Reddien et al., 2005a,b; Rouhana et al., 2010; Salvetti et al., 2005; Wang et al., 2007; Wang et al., 2010), signals that stimulate growth or degrowth of $S$. mediterranea, and how those gross changes are coordinated with stem cell activity remain unknown (Pellettieri and Sánchez Alvarado, 2007).

In this study, we examine ilp-1, a neuroendocrine insulin-like peptide (Collins etal., 2010) and inr-1, a putative insulin-like peptide receptor tyrosine kinase in $S$. mediterranea. We outline a regulatory role for the insulin/IGF signaling pathway in controlling adult size homeostasis and describe correlative changes in proliferating cell populations. Our data suggest that insulin/IGF signaling differentially regulates adult stem cell populations based on nutrient availability.

\section{Results}

\section{An insulin-like peptide is expressed in the nervous system and testis lobes}

To determine how an organism that can grow and degrow as an adult (Pellettieri and Sánchez Alvarado, 2007) regulates its size, we explored insulin/IGF signaling as a candidate pathway in S. mediterranea. In a genome-wide analysis of $S$. mediterranea neuropeptides, Collins et al., (2010) reported a putative insulin-like peptide, smed-ilp-1 (hereafter referred to as ilp-1). We examined the sequence of ilp-1 and verified the presence of six conserved cysteine residues, which are known to contribute to the tertiary structure of the peptide (Fig. 1 A,B; Chan and Steiner, 2000; Smit et al., 1998). The relatively low degree of conservation shows that ilp-1 is diverged from its invertebrate counterparts, which is consistent with the current perspective that insulin-like peptide sequences are highly diverged across invertebrate species (Chan and Steiner, 2000; De Meyts, 2004). Additionally, we identified a single predicted protein with similarity to an insulin/IGF receptor tyrosine kinase, smed-inr-1 (hereafter referred to as inr-1; Fig. 1 C,D).

According to northern blot analyses, the full-length mRNA sequence encoded by ilp-1 is approximately 500 nucleotides (Fig. 1E), while inr-1 mRNA is approximately 4000 nucleotides (Fig. 1F). To obtain full-length sequence for both ilp-1 and inr-1 mRNAs, we performed 5' and 3' RACE. For ilp-1, we obtained a full-length cDNA sequence of 627 nucleotides, which includes a 143-codon open reading frame. For inr-1, we obtained a full-length cDNA sequence of 4648 nucleotides that includes a 1391-codon open reading frame.

Northern blot analyses also revealed that $i l p-1$ was expressed at higher levels in the sexually reproducing strain (Fig. 1E). We validated this observation by performing reverse-transcriptase quantitative PCR (qPCR) and found a 3.2-fold difference in ilp-1 expression between asexual and sexual animals $(p<0.05)$. No significant difference was observed in expression for inr- 1 between the strains by either northern blot (Fig. 1F) or qPCR.

The increased expression of ilp-1 in the sexual strain suggested that insulin signaling might play a role in sexual reproduction for $S$. mediterranea. To examine which tissues are associated with insulin expression, we performed in situ hybridization on both the sexual and asexual strains of the animal. ilp-1 expression was detected in cells within the central nervous system (cephalic ganglia and nerve cords) in both sexual and asexual animals (Fig. 2 A-C). In the sexual strain, ilp-1 expression was also detected in the testes lobes (Fig. 2A); the abundance of signal in more luminal regions

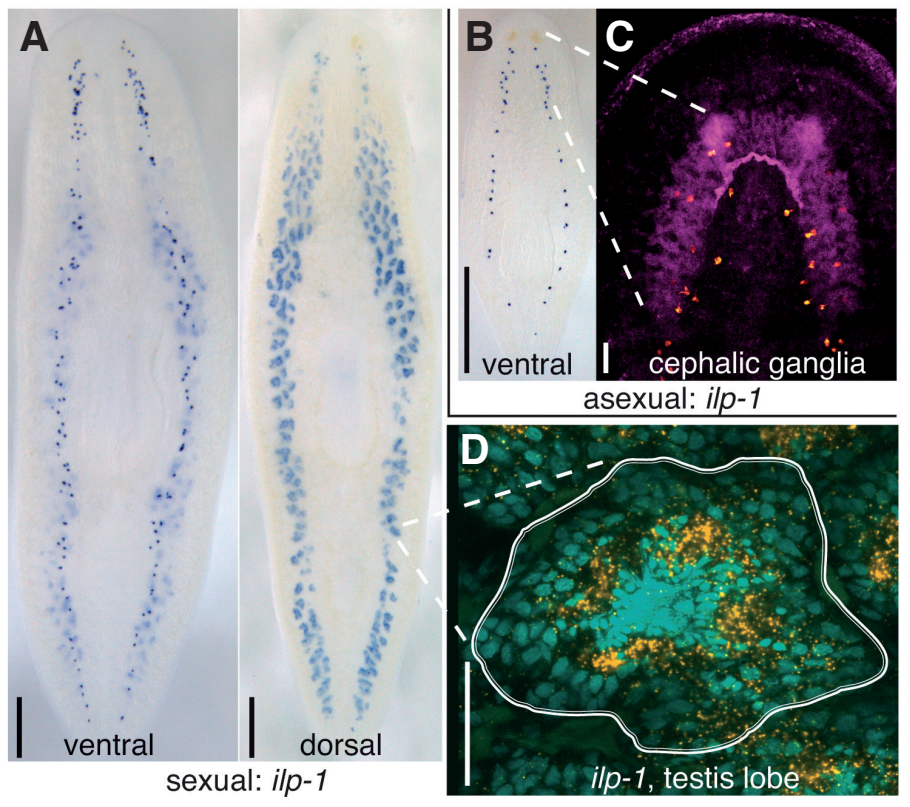

Fig. 2. Expression patterns of ilp-1 and inr-1 visualized by whole-mount in situ hybridization. (A) Ventral view of a sexual planarian shows ilp-1 expression in the cephalic ganglia and nerve cords; dorsal view reveals ilp-1 expression in testis lobes. (B) ilp-1 expression in an asexual planarian. (C) ilp-1 (orange) expression in an asexual planarian co-stained with neural markers anti-phospho-tyrosine and VC-1 (magenta). (D) A testis lobe with ilp-1 (orange) expression and the nuclear stain DAPI (cyan). Scale bars: 0.5 mm (black; $A, B$ ) or 50 um (white; $C, D$ ).

(Fig. 2D) suggests that ilp-1 is expressed in spermatocytes and spermatids (Wang et al., 2010). The expression of ilp-1 in developing and mature testes implies that, in $S$. mediterranea, insulin may play a paracrine or autocrine role distinct from neural regulation in supporting testis growth and spermatogenesis. inr-1 appears to be expressed at low levels throughout the animal, making it difficult to determine definitively by in situ hybridization which cell types express this gene.

\section{Neurally expressed insulin maintains animal size}

Nutritional intake and insulin-like peptides moderate growth in developing organisms (Ikeya et al., 2002; Tatar et al., 2003). However, little is known about how these factors act on adult stem cell populations in vivo. The ability of adult $S$. mediterranea to grow and degrow allows a gross assessment of adult stem cell function after post-development disruption of insulin/IGF signaling.

To test if insulin/IGF signaling in $S$. mediterranea might be responsible for regulating adult size homeostasis, we created dsRNA feeding vectors for ilp-1 and inr-1. Then, according to established RNA interference (RNAi) protocols (Newmark et al., 2003), we fed bacteria expressing dsRNA to large, sexually reproducing S. mediterranea on a weekly basis. Aschedule of weekly feeding promotes growth or maintenance of animal size. Over the course of these feedings, ilp-1(RNAi) and inr-1(RNAi) animals degrew to $90 \%$ and $70 \%$ respectively of the control cohort mean size, despite eating normally (Fig. $3 \mathrm{~A}, \mathrm{~B} ; \mathrm{p}<0.01$ ). Animals with both genes knocked down simultaneously had a phenotype indistinguishable from the inr-1(RNAi) cohort, which is consistent with ilp-1 and inr-1 acting through a similar mechanism to control animal size. To further assess the possibility that ilp-1 and inr-1 act through a similar 
mechanism, we performed qPCR on animals after RNAi. After four weeks, inr-1 (RNAi) animals had an approximately 10-fold increase in ilp-1 expression $(p<0.01)$, which is consistent with a feedback mechanism between these two members of the insulin pathway. No change in inr-1 expression was detected in ilp-1(RNAi) animals.

In S. mediterranea, animals starved for longer than a few weeks noticeably shrink (Newmark and Sánchez Alvarado, 2002). To examine how disruption of insulin signaling compared to lack of nutritional intake, we compared insulin-disrupted animals to starved animals. After eight weeks of RNAi, the phenotypes resulting from disrupted insulin signaling are, on a gross level, similar to those of nutritional deprivation (Fig. 3 A,B). However, in the sexual strain of S. mediterranea, there are two distinct populations of cells producing insulin-like peptides: neuroendocrine cells and gonadal cells. To examine whether the neuroendocrine ilp-1 signaling is necessary for maintaining adult tissue homeostasis and size, we repeated the degrowth experiments with a cohort of asexual animals. Over the course of 4 weeks, we observed that animals with disrupted insulin/IGF signaling were 5 to $10 \%$ smaller than control animals (inr-1(RNAi) $=\mathrm{p}<0.05$; data not shown), consistent with the response of sexual animals at this time point (Fig. 3A). Therefore, we demonstrate that regulation of adult size homeostasis in $S$. mediterranea is dependent not only on nutritional status, but also on neuroendocrine ilp-1 signaling. We also show that, on a gross level, the phenotypes of disrupted insulin signaling and nutritional deprivation are similar.

\section{Disruption of insulin signaling affects stem cell proliferation}

When S. mediterranea degrow after lack of nutritional intake, there is a correlative increase in apoptotic activity (Pellettieri et al., 2010) but no significant change in individual cell size (Pellettieri and Sánchez Alvarado, 2007; Romero and Baguñá, 1991). However, changes in overall animal size could reflect not only changes
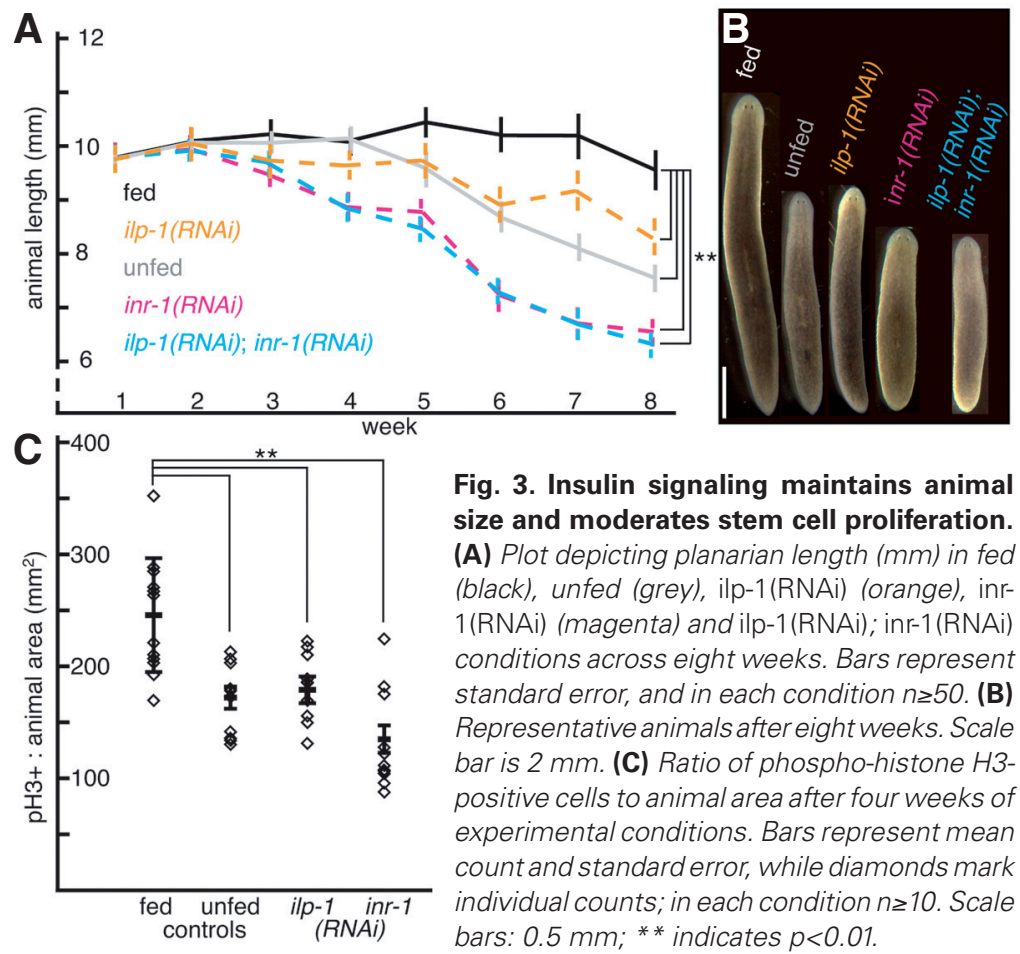

Fig. 3. Insulin signaling maintains animal size and moderates stem cell proliferation. (A) Plot depicting planarian length $(\mathrm{mm})$ in fed (black), unfed (grey), ilp-1(RNAi) (orange), inr1(RNAi) (magenta) and ilp-1(RNAi); inr-1 (RNAi) conditions across eight weeks. Bars represent standard error, and in each condition $n \geq 50$. (B) Representative animals after eight weeks. Scale bar is $2 \mathrm{~mm}$. (C) Ratio of phospho-histone H3positive cells to animal area after four weeks of experimental conditions. Bars represent mean count and standard error, while diamonds mark individual counts; in each condition $n \geq 10$. Scale bars: $0.5 \mathrm{~mm} ;{ }^{*}$ indicates $p<0.01$. in overall cell survival, but also altered stem cell dynamics. In asexual $S$. mediterranea, neoblasts are the only mitotically active somatic cells, and mitotic neoblasts can be labeled specifically with anti-phosphohistone H3 (Newmark and Sánchez Alvarado, 2000). Therefore, to explore whether or not adult stem cell activity changes are correlative with changes in adult size, we examined mitotic activity in asexual animals after nutritional deprivation or disruption of insulin signaling.

Animals with no nutritional intake over four weeks showed approximately $30 \%$ fewer mitotic cells in comparison to controls (Fig. 3C; $\mathrm{p}<0.01$ ). This finding correlates an overall decrease of proliferative neoblasts with a decrease in animal size. The decrease in total proliferative neoblasts could arise from a decrease in total neoblasts with a constant rate of proliferation and/or a decrease in rate of proliferation across a constant population of neoblasts. Earlier work supports a scenario in which the percentage of neoblasts within the total cell population remains roughly constant during growth and degrowth due to lack of nutrition (Baguñá and Romero, 1981). To distinguish between these two possible scenarios, we performed qPCR on two piwihomologues preferentially expressed in neoblasts: smedwi-1 and smedwi-2 (Guo et al., 2006; Reddien et al., 2005b). We did not detect a significant difference in smedwi-1 or smedwi-2 relative expression levels (normalized to ubiquitously-expressed $b$-tubulin) between fed or starved animals (data not shown). These data are consistent with the maintenance of a roughly proportional population of neoblasts as observed by Baguñá and Romero (1981). Though subtle changes in the total neoblast population may still be present, they are not equivalent to the approximately $30 \%$ decrease of mitotic cells (Fig. 3C). Therefore, the observed decrease in mitotic cells likely results in part from a decrease in cell proliferation rate.

With fed and starved animals as baselines, we examined proferation in animals with disrupted insulin signaling. We found that ilp-1(RNAi) or inr-1(RNAi) animals, like starved animals, had approximately $30 \%$ and $50 \%$, respectively, decreased proportions of mitotic cells compared to fed animals (Fig. $3 \mathrm{C} ; \mathrm{p}<0.01)$. ilp-1(RNAi) animals were similar in size to starved animals, and the proportion of proliferative cells was indistinguishable across these two conditions. inr-1(RNAi) animals, which after eight weeks were smaller compared to starved animals, also had a smaller proportion of mitotically active cells (Fig. $3 \mathrm{C} ; \mathrm{p}<0.05$ ).

As with the unfed cohort, there was no difference in relative expression of either smedwi-1 or smedwi-2 in the ilp-1(RNAi) or inr-1(RNAi) conditions via qPCR. Thus, it appears that animal size, in conditions of nutritional deprivation or disrupted insulin signaling, is regulated at least in part by decreasing the rate of proliferation across a population of neoblasts. Furthermore, the mechanism by which starved animals and insulin-disrupted animals degrow appears to be similar in terms of changes in adult stem cell proliferation.

\section{Disruption of insulin signaling impairs testis growth and spermatogenesis}

As sexually mature $S$. mediterranea degrow past a certain point, their gonads regress (Newmark and Sánchez Alvarado, 2002). Thus, it follows that size and nutritional status are factors in gonad development and maintenance. Nutritional deprivation and disruption of insulin signaling both result in 

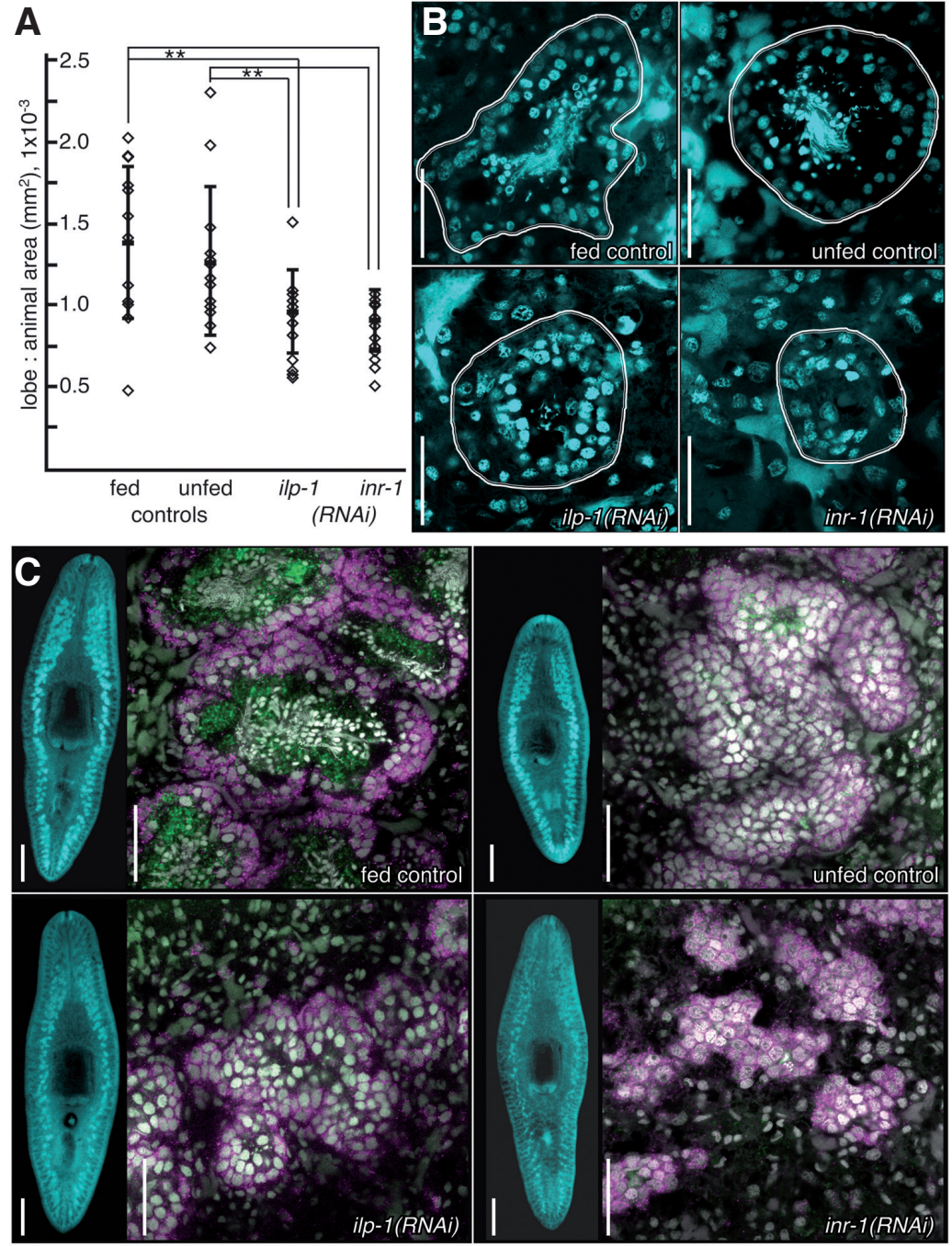

Fig. 4. Insulin signaling maintains size of testis lobes and supports spermatogenesis. (A) Plot depicting the ratio of testis lobe area to animal area in $\mathrm{S}$. mediterranea after RNAi for four weeks. Bars represent mean size and standard error, while diamonds mark the average area of five lobes in one worm; in each condition $n \geq 10$. (B) Representative testis lobes stained with nuclear maker DAPI (cyan), with the area outlined in white. Scale bars are $50 \mu \mathrm{m}$. (C) Representative whole animals with testis lobes visualized with nuclear marker DAPI (cyan); each whole animal is next to a corresponding testis lobe after in situ hybridization to detect germinal histone H4 (magenta) and immunostaining with anti-Tral (green). Scale bars: 50 um for testis lobes $(B, C)$ and $0.5 \mathrm{~mm}$ for whole animals $(C)$; * indicates $p<0.05$.

decreased size and correlatively decreased neoblast proliferation (Fig. $3 \mathrm{~A}-\mathrm{C}$ ). If insulin signaling affects the neoblast population in S. mediterranea, it may also moderate germline stem cell proliferation. Consistent with this hypothesis, ilp-1 is expressed within testis lobes (Fig. 2A,D,E). To explore whether insulin signaling influences germline dynamics, we examined testis lobe size and morphology after nutritional deprivation or disruption of insulin signaling.

In sexually mature $S$. mediterranea that were starved for four weeks, we found that, in the size range of animals observed, there was actually no proportional decrease in lobe size compared to control animals (Fig. $4 \mathrm{~A}, \mathrm{~B}$ ), although the starved population was smaller than control animals (Fig. $4 \mathrm{C} ; \mathrm{p}<0.05$ ). Correlating with the overall lobe size decrease in the smaller starved population, there were fewer spermatids, as visualized by the nuclear stain, DAPI (spermatids were observed in: 4/12 starved animals; $11 / 12$ control animals). We therefore performed in situ hybridization to detect germinal histone H4 RNA, which is expressed in neoblasts as well as in spermatogonia and spermatocytes (Wang et al., 2007). We co-labeled with anti-Trailer-hitch antibodies that recognize RAP55 protein expressed in spermatocytes and spermatids (Fig. 4C; see Wang et al., 2010). We detected RAP55 protein in the luminal regions of testis lobes when spermatids were visible in both fed and starved animals.

After establishing that testis lobe size in mature animals appears to be similarly proportional to animal size across normally fed and starved animals, we examined lobe sizes in animals after ilp-1(RNAi) or inr-1(RNAi) treatment. In these conditions, testis lobes were proportionally smaller by approximately $30 \%$ and $35 \%$, respectively, in comparison to the control cohort (Fig. $5 \mathrm{~A}-\mathrm{C} ; \mathrm{p}<0.05$ ). Correlated with the decreased lobe size, we also observed a decrease in maturing spermatids (5/12 ilp-1(RNAi) animals; 4/12 inr$1(R N A i)$ animals). We observed testis lobes with luminal cavities, as if there were room for maturing spermatids, but some of these hollows lacked the condensed nuclei/ RAP55-positive indicators of cells progressing through spermatogenesis; the inr-1(RNAi) animals generally appeared to have a more severe phenotype (Fig. 4C). Thus, insulin signaling is not necessary for the maintenance, per se, of spermatogonia in testes lobes, because $\mathrm{gH} 4$-positive cells are present in both ilp-1(RNAi) and inr-1(RNAi) animals. Rather, insulin-like signaling appears to be involved in the subsequent amplification divisions and/or differentiation of these cells.

\section{Discussion}

In S. mediterranea, neuroendocrine ilp-1 is involved in regulation of growth and tissue homeostasis, consistent with the conserved developmental functions of insulin signaling in other species (Chan and Steiner, 2000; De Meyts, 2004). S. mediterranea has a plastic adult size and its adult stem cell population is readily observable by modern molecular techniques (Newmark and SánchezAlvarado, 2002). Thus, the planarian provides a unique perspective on how insulin signaling regulates proliferating stem and germline cells in an adult organism

We observed that after disrupting insulin signaling via RNAi, animals degrew but otherwise remained healthy on a gross level. In contrast, RNAi knockdown of genes that are required for proper neoblast function as well as lethal irradiation (which causes loss of the neoblast population), result in a distinct phenotype: animals without neoblasts fail to regenerate, curl up ventrally, and die on the order of days to weeks (Eisenhoffer et al., 2008; Guo et al., 2006; Hayashi et al., 2006; Reddien et al., 2005a,b; Rouhana et al., 2010; Salvetti et al., 2005). Thus, under conditions of nutritional deprivation or disrupted insulin signaling, a basal rate of stem cell proliferation is maintained, allowing the organism to survive in the chance that more nutrition will become available. Insulin signaling in S. mediterranea is not necessary for immediate survival/health of the organism or stem cells, but acts on a longer 
time scale to coordinate nutrition, adult tissue homeostasis, and stem cell dynamics.

The effects of inr-1(RNAi) on animal size, mitotic index, and spermatogenesis are consistently more severe than those of ilp-1(RNAi) alone. These data suggest that there is complexity in $S$. mediterranea insulin signaling that is unaccounted for by considering only these two genes. Given the degree of primary sequence divergence in insulin-like peptides (Chan and Steiner, 2000) and the fact that there are many organisms with more than one insulin-like peptide (Daftary and Gore, 2005; Wu and Brown, 2006), it seems likely that there are other currently unidentified insulin-like peptides in S. mediterranea. Additional members in the insulin signaling pathway would contribute to greater complexity and control of the pathway in response to distinct environmental cues.

We show that insulin signaling moderates not only neoblast proliferation, but also testis lobe maintenance. In D. melanogaster and C. elegans, nutritional deprivation or insulin signaling disruption leads to a decrease in germline stem cell proliferation in gonads (Drummond-Barbosa and Spradling, 2001; McLeod et al., 2010; Michaelson et al., 2010). Ovarian germ cell proliferation is regulated specifically by neurally produced insulin-like peptides (LaFever and Drummond-Barbosa, 2005). In S. mediterranea, ilp-1 is expressed in developed testis lobes. Similarly, leydig cells in mammalian testes express an insulin-like factor (InsI3), however the effects of Insl3 on germ cell maintenance and spermatogenesis are yet to be understood (Ivell et al., 2005). S. mediterranea provides an opportunity to study another organism with a testis-derived insulin-like peptide, which has implications for understanding spermatogenesis and causes of infertility in mammals.

In S. mediterranea, we show that insulin signaling and nutritional deprivation regulate both adult stem cells and germline cells. However, disruption of insulin signaling has a more severe effect on the germline than starvation does (Fig. 4 A-C). These data imply that insulin signaling is not only a possible mechanism for the communication of nutritional status, but also has a separate role as a growth factor. Furthermore, these data demonstrate the complexity and variety of tissue responses to a single environmental cue. During times of starvation, animals may initially favor maintenance of the germline and potential fertility, selectively suppressing proliferation of other adult stem cell populations to compensate for the lack of nutritional intake. Exploration of the transcriptional changes in neoblasts and germline cells after starvation or insulin signaling disruption would yield clues as to how these cell populations respond differently to an environmental challenge.

Our observations contribute to a greater understanding of the roles of insulin family signaling and nutrition in stem cell dynamics. We show that insulin signaling moderates both adult stem cell and germline cell populations in vivo. Our findings suggest that specific stem cell populations are differentially regulated depending upon an organism's perceived resources. Intriguingly, insulin family signaling is also disrupted in many cancers (Maki, 2010; Samani et al., 2007). Consistently, in S. mediterranea, we show that decreased insulin/IGF signaling decreases neoblast proliferation while Oviedo et al., (2008) induced cancer-like growths by knockdown of PTEN, anAKT pathway (insulin-signaling) inhibitor. A better understanding of adult stem cell dynamics after alterations in insulin signaling may influence treatments for both infertility and cancer. Further analysis of the roles of insulin signaling and nutrition in the adult stem cell and germline populations of $S$. mediterranea may offer insight into organismal controls of cell proliferation and differentiation.

\section{Materials and Methods}

\section{Animal culture}

Clonal asexual and sexual (hermaphroditic) strains of Schmidtea mediterranea were housed at $20^{\circ} \mathrm{C}$ in $1.0 \mathrm{x}$ and $0.75 \mathrm{x}$ Montjuïc salts, respectively (Cebrià and Newmark, 2005). Animals were fed ground organic calf liver (Vantage USA, Lenoir, NC) once per week. Prior to fixation for imaging, animals were starved for at least one week to reduce background and increase animal integrity during processing.

For growth/degrowth experiments, we used three biological replicate groups of 15 to 20 sexual $S$. mediterranea per condition, leading to a total of at least 50 animals per condition per time point. We replicated the findings in asexual animals using one biological group of at least 20 animals per condition per time point. Live images of animals were taken with a Leica DFC420 camera mounted on a Leica M205A stereomicroscope (Leica, Wetzlar, Germany), utilizing LAS3.6 software. To accurately and consistently capture images of animal length/size, animals were accustomed to a Petri dish for a minimum of 5 minutes prior to imaging. $S$. mediterranea displays negative phototactic behavior. Thus, just prior to imaging, an LED light source was turned on, causing animals to extend as they moved away from the light. For the first time point, both animal length and area were calculated; they were found to be proportional and length was used as a proxy for area/ size thereafter. Mean animal length and standard error were calculated for each graphed experimental condition. For the final experimental timepoint, differences in length between all conditions were analyzed for significance via ANOVA, followed by Tukey's HSD post-hoc test.

\section{Gene identification}

To identify insulin and insulin receptor homologues in S. mediterranea, EST (Zayas et al., 2005) and genomic resources (Robb et al., 2007) were searched via BLAST with query sequences from other species including Bombyx mori, C. elegans, D. melanogaster, Homo sapiens and Schistosoma mansoni (NCBI). Insulin identity depends on the presence of six canonical cysteine residues and a signal sequence (Chan and Steiner, 2000, Smit et al., 1998). After this bioinformatic search, only one putative insulin-like peptide gene (ilp-1) encoded a protein with all six cysteines at the proper location in gene sequence as well as a putative signal sequence (SignalP 3.0; (Bendtsen etal., 2004)). Agene encoding for a candidate insulin receptor (inr-1) was identified based on similarities with other insulin receptor tyrosine kinases. inr-1 had both ligand-binding ( $\mathrm{L}$ ) and tyrosine kinase domains, as well as other conserved domains consistent with insulin receptor tyrosine kinases (SMART 6 protein domain predictor; (Letunic et al., 2009; Schultz et al., 1998)). ESTs with partial sequence, in the pBluescript II (SK+) vector, were available for both ilp-1 (PL05016A1H10) and inr-1 (PL05016A2F11) from our EST collection (Zayas et al., 2005). EST matches were verified by sequencing prior to use as template for further experiments.

Full-length sequences for ilp-1 and inr-1 were obtained as follows: RNA was extracted from the sexual strain of $S$. mediterranea (Trizol, Invitrogen, Carlsbad, CA), DNase digested (DNA-free RNA Kit, Zymo Research, Orange, CA), oligo (dT) purified (Poly-A Purist Kit, Ambion, Austin, TX), then used as template for cDNA synthesis (iScript cDNA Synthesis Kit, Bio-Rad, Hercules, CA). Both 5' and 3' RACE (First Choice RLM RACE Kit, Ambion, Austin, TX) were performed with nested, gene-specific primers. Due to the presence of AT-rich regions in the insulin receptor that caused false 3' poly-A ends, this process was repeated until all receptor domains were identified. Accession numbers: Smed-ilp-1, BK007034; Smed-inr-1, JN049497.

Riboprobe Synthesis, Northern Blot, In situ Hybridization. Riboprobes were synthesized for use in northern blot and in situ hybridization. In vitro transcription using T3 polymerase was performed with partial cDNA sequence template for ilp-1 and inr-1. Probes were synthesized with Digoxigenin-12-UTP (Roch, Mannheim, Germany), Fluorescein-12-UTP (Roche, Mannheim, Germany) or Dinitrophenol-11-UTP (Perkin Elmer, Waltham, MA). Additionally, because the original inr-1 partial cDNA clone corresponded to only 762 of 4654 total nucleotides, two alternate probes 
to unique 5' and 3' regions were transcribed from nested PCR-amplified product (Platinum Taq, Invitrogen, Carlsbad, CA) using gene-specific primers flanked by T3 or T7 promoter sites. All three inr-1 probes were tested side by side in northern blot and in situ hybridization to ensure that they all yielded similar results. The primers used were:

inr-1 alternate probe sequences, 5' and 3' of EST sequence:

inr-1 5' forward: 5'-CGGTGGGGAAAATTTGCAGAAA-3';

inr-15' reverse: 5'-CAGAACCTTCCAAAATCGTGA-3';

inr-1 3' forward: 5'-CGCTCTGGGCCATACAAATTGC-3';

inr-13' reverse: 5'-TGGGTTTATTCATTGACTTTCC-3'

The flanking $\mathrm{T} 7$ and $\mathrm{T} 3$ polymerase promoter sequences were:

T7: 5'-GTAATACGACTCACTATAGGG-3';

T3: 5'-CAATT AACCCTCACTAAAGGG-3'

Northern blots were performed according to standard protocol (Sambrook and Russell, 2001), utilizing digoxigenin probes (above), $\alpha$-digoxigenin AP antibody (Roche, Mannheim, Germany) and chemiluminescence (CDPSTAR, Roche, Mannheim, Germany). Luminescent blots were visualized with a FluoroChem Q (Alpha Innotech, San Leandro, CA).

In situ hybridization was performed according to a standard protocol for asexual and sexual animals (Collins et al., 2010; Pearson et al., 2009). Chromogenic visualization utilized digoxigenin-labeled riboprobes and $\alpha$-digoxigenin-AP antibody previously described and was developed with NBT-BCIP (SigmaFast BCIP/NBT, Sigma, St. Louis, MO) in high salt AP buffer (100mM Tris, pH 9.5; $100 \mathrm{mM} \mathrm{NaCl} ; 50 \mathrm{mM} \mathrm{MgCl}_{2} ; 0.1 \%$ tween-20) with 10\% PVA. After development, animals were post-fixed (4\% FA in PBS $+1 \%$ Triton $X-100$ ) for 15 minutes, washed in $100 \%$ ethanol $2 \times 10$ minutes, returned to PBS $+1 \%$ Triton X-100, then cleared and mounted in $80 \%$ glycerol. Animals developed chromogenically were imaged over a piece of white filter paper with the Leica microscope, camera, and software described above.

Fluorescent in situ hybridization utilized all three probe types, coupled with $\alpha$-digoxigenin-POD (Roche, Mannheim, Germany), $\alpha$-fluorescein-POD (Roche, Mannheim, Germany) or $\alpha$-dinitrophenol-HRP (Perkin Elmer, Waltham, MA). Prior to development, animals were placed in TNT buffer (100mM Tris, pH7.5; $150 \mathrm{mM} \mathrm{NaCl}$; 0.05\% tween-20) for 10 minutes. FISH was developed with Сy3-, Cy5- or fluorescein-tyramide TSA-Plus kits (Perkin Elmer, Waltham, MA), and if multiple probes were utilized, then peroxidase reactions were quenched (1 hour incubation with $1 \% \mathrm{H}_{2} \mathrm{O}_{2}$ in TNT buffer) prior to detection of another probe. Samples were co-stained with the nuclear marker DAPI (Sigma-Aldrich, St. Louis, MO). Samples were mounted in Vectashield (Vector Laboratories, Burlingame, CA) and imaged on a Zeiss LSM 710 confocal microscope (Carl Zeiss, Germany) with Zen 2008 software (Carl Zeiss, Germany) or a Zeiss SteREO Lumar.V12 microscope. Further analysis, including calculation of lobe size or manual numbering/counting of cells, was also carried out with Zen 2008 software.

Immunofluorescence. Staining with anti-phosphohistone H3 and DAPI were performed with animals killed in $5 \%$ n-acetyl cysteine for 5 minutes, rinsed once in PBS, then fixed for 20 minutes in $4 \%$ formaldehyde in PBSTx (PBS $+0.3 \%$ Triton X-100). Animals were rinsed in PBSTx for $3 x$ 5 minutes, then were bleached overnight in approximately $6 \% \mathrm{H}_{2} \mathrm{O}_{2}$ in PBSTx. Animals were rinsed again in PBSTx for $3 \times 5$ minutes, blocked at room temperature for 4 hours in PBSTx $+0.6 \%$ BSA $+0.4 \%$ fish gelatin, and exposed to a 1:100 dilution of anti-mouse phospho-histone $\mathrm{H} 3$ serine 10 (05-806, Millipore, Temecula, CA) in PBSTx at $4^{\circ} \mathrm{C}$ overnight. After washing 6 times over a minimum of 4 hours, animals were exposed to the secondary Goat anti-Mouse POD (Pierce/Thermo Fisher Scientific, Rockford, IL) for either 4 hours at room temperature or $4^{\circ} \mathrm{C}$ overnight. The animals were then washed in PBSTx 6 times in 1 hour, placed into TNT buffer (above) for 10 minutes, then developed with the Perkin-Elmer TSA kit (Perkin Elmer, Waltham, MA). Staining with anti-phospho-tyrosine and VC-1 was performed as previously described (Cebrià and Newmark, 2005) after in situ hybridization. Staining with anti-Tral was performed after in situ hybridization as previously described (Wang et al., 2010).

\section{RNA interference}

EST sequences for insulin and insulin receptor were cloned into the
pPR244 plasmid (Reddien et al., 2005a) via the Gateway BP Clonase Kit (Invitrogen, Carlsbad, CA). Plasmids were transformed into RNasellldeficient HT115(DE3), which expresses T7 polymerase after induction with IPTG (Timmons et al., 2001) thus producing dsRNA. Single colonies were selected for sequencing to confirm successful cloning, and cultures were grown and induced as previously described (Newmark et al., 2003). Control vector was empty pPR242, transformed into HT115(DE3). For feeding, a pellet of cells from $10 \mathrm{ml}$ of induced culture, stored at $-80^{\circ} \mathrm{C}$, was thawed, mixed with 60 ul of liver homogenate, then administered to animals. There was an excess of food in all RNAi/feeding experiments so animals could eat to satiety. Animals undergoing RNAi were fed once per week, then fixed and processed as described above. For experiments involving pH3-positive cell counts as well as experiments involving analysis of testis lobes, a minimum of 10 animals were used in each condition. Differences between conditions were analyzed for significance via ANOVA, followed by Tukey's HSD post-hoc test.

rt-qPCR. Each reverse-transcriptase quantitative PCR (qPCR) experiment described was performed on three biological replicate samples of 2-3 worms each. RNA from each sample was extracted with Trizol (Invitrogen, Carlsbad, CA), DNase digested (DNA-free RNA Kit, Zymo Research, Orange, CA), then used as template for cDNA synthesis (iScript cDNA Synthesis Kit, Bio-Rad, Hercules, CA). cDNA was diluted to $100 \mathrm{ng} / \mathrm{ul}$ prior to experimentation.

Samples were run in experimental triplicate. qPCR was performed with GoTaq Mastermix (Promega, Madison, WI) on a StepOnePlus real-time PCR system (Applied Biosystems, Carlsbad, CA) with StepOne Software v2.1 according to the $\Delta \Delta C$ C protocol with $b$-tubulin (PL05008A2H07) as a normalization control. Statistical analysis was performed using a Student's $t$-test on $\triangle \mathrm{CT}$ values. The primers used were:

$\beta$-tubulin forward: TGGCTGCTTGTGATCCAAGA

$\beta$-tubulin reverse: AAATTGCCGCAACAGTCAAATA

ilp-1 forward: CTAAGACACTTTTTCGCCAATCG

ilp-1 reverse: $\quad$ TTTGTAAATCGGGTGCATTATGTTA

inr-1 forward: TGGAAACCAGAACCAAGGAG

inr-1 reverse: $\quad$ CATGACTCCATGCACTTGTCA

smedwi-1 forward: GAAGAGCTGGGGGATGTGTA

smedwi-1 reverse: TTCACGACCACGAATCGTAA

smedwi-2 forward: GGCGATCAACCATTTGTTCT

smedwi-2 reverse: CTTTCTCGACGCATTGGTTT

\section{Acknowledgements}

We thank all Newmark Lab members for support and advice, and Jim Collins, Rachel Roberts-Galbraith, Labib Rouhana, and James Sikes for critical feedback on this manuscript. We thank Brad Kubick for the original identification of candidate insulin-like genes and Jim Wilhelm for the anti-Tral antibodies. We apologize to our colleagues whose work we were unable to cite due to space limitations. This work was supported by NSF (IOS-0744689) and NIH (R01-HD043403). P.A.N. is an Investigator of the Howard Hughes Medical Institute.

\section{References}

BAGUÑ́, J. and ROMERO, R. (1981). Quantitative analysis of cell types during growth, degrowth and regeneration in the planarians Dugesia mediterranea and Dugesia tigrina Hydrobiologia 84: 181-194.

BAGUÑ́, J., SALO, E. and AULADELL, C. (1989). Regeneration and pattern formation in planarians. III. Evidence that neoblasts are totipotent stem cells and the source of blastema cells. Development 107: 77-86.

BENDTSEN, J., NIELSON, H., VON HEIJNE, G. and BRUNAK, S. (2004). Improved prediction of signal peptides: SignalP 3.0. J Mol Biol 340: 783-795

BRØNDSTED, H. (1969). Planarian Regeneration. Pergamon, London.

CEBRIÀ, F. and NEWMARK, P. (2005). Planarian homologs of netrin and netrin receptor are required for proper regeneration of the central nervous system and the maintenance of nervous system architecture. Development 132: 3691-3703.

CHAN, S. and STEINER, D. (2000). Insulin through the ages: Phylogeny of a growth 
promoting and metabolic regulatory hormone. Am Zool 40: 213-222.

CHEN, C., JACK, J. and GAROFALO, R. (1996). The Drosophila insulin receptor is required for normal growth. Endocrinology 137: 846-856.

COLLINS, J.R., HOU, X., ROMANOVA, E., LAMBRUS, B., MILLER, C., SABERI, A., SWEEDLER, J. and NEWMARK, P. (2010). Genome-wide analyses reveal a role for peptide hormones in planarian germline development. PLoS Bio/8: e1000509.

DAFTARY, S. and GORE, A. (2005). IGF-1 in the brain as a regulator of reproductive neuroendocrine function. Exp Biol Med 230: 292-306.

DE MEYTS, P. (2004). Insulin and its receptor: structure, function and evolution. BioEssays 26: 1351-1362.

DRUMMOND-BARBOSA, D. and SPRADLING, A.C. (2001). Stem cells and their progeny respond to nutritional changes during Drosophila oogenesis. Dev Biol 231: $265-278$.

EISENHOFFER, G., KANG, H. and SÁNCHEZ ALVARADO, A. (2008). Molecular analysis of stem cells and their descendants during cell turnover and regeneration in the planarian Schmidtea mediterranea. Cell Stem Cell 3: 327-339.

GUO, T., PETERS, A. and NEWMARK, P. (2006). A Bruno-like gene is required for stem cell maintenance in planarians. Dev Cell 11: 159-169.

HAYASHI, T., ASAMI, M., HIGUCHI, S., SHIBATA, N. and AGATA, K. (2006). Isolation of planarian X-ray-sensitive stem cells by fluorescence-activated cell sorting. Dev Growth Differ 48: 371-380.

HSU, H.-J. and DRUMMOND-BARBOSA, D. (2009). Insulin levels control female germline stem cell maintenance via the niche in Drosophila. Proc. Natl. Acad. Sci. USA 106: 1117-1121

IKEYA, T., GALIC, M., BELAWAT, P., NAIRZ, K. and HAFEN, E. (2002). Nutrientdependent expression of insulin-like peptides from neuroendocrine cells in the CNS contributes to growth regulation in Drosophila. Curr Biol 12: 1293-1300.

IVELL, R., HARTUNG, S. and ANAND-IVELL, R. (2005). Insulin-like factor 3: Where are we now? Ann N Y Acad Sci 1041: 486-496.

LAFEVER, L. and DRUMMOND-BARBOSA, D. (2005). Direct control of germline stem cell division and cyst growth by neural insulin in Drosophila. Science 309 1071-1073.

LETUNIC, I., DOERKS, T. and BORK, P. (2009). SMART 6: recent updates and new developments. Nucleic Acids Res 37: 229-232.

MAKI, R. (2010). Small is beautiful: Insulin-like growth factors and their role in growth, development, and cancer. J Clin Oncol 28: 4985-4995.

MCLEOD, C., WANG, L., WONG, C. and JONES, D. (2010). Stem cell dynamics in response to nutrient availability. Curr Biol 20: 2100-2105.

MICHAELSON, D., KORTA, D., CAPUA, Y. and HUBBARD, E. (2010). Insulin signaling promotes germline proliferation in C. elegans. Development 137: 671-680.

MORGAN, T. (1902). Growth and regeneration in Planaria lugubris. Arch Ent Mech Org 13: 179-212.

NEWMARK, P., REDDIEN, P., CEBRIÀ, F. and SÁNCHEZ ALVARADO, A. (2003). Ingestion of bacterially expressed double-stranded RNA inhibits gene expression in planarians. Proc. Natl. Acad. Sci. USA 100: 11861-11865.

NEWMARK, P. and SÁNCHEZ ALVARADO, A. (2002). Not your father's planarian: A classic model enters the era of functional genomics. Nat Rev Genet 3: 210-219.

OVIEDO, N., NEWMARK, P. and SÁNCHEZALVARADO, A. (2003). Allometric scaling and proportion regulation in the freshwater planarian Schmidtea Mediterranea. Dev Dyn 226: 326-333

OVIEDO, N., PEARSON, B., LEVIN, M. and SÁNCHEZ ALVARADO, A. (2008). Planarian PTEN homologs regulate stem cells and regeneration through TOR signaling. Disease Mod. Mech. 1: 131-143.

PEARSON, B., EISENHOFFER, G., GURLEY, K., RINK, J., MILLER, D. and SÁNCHEZ ALVARADO, A. (2009). Formaldehyde-based whole-mount in situ hybridization method for planarians. Dev Dyn 238: 443-450.

PELLETTIERI, J., FITZGERALD, P., WATANABE, S., MANCUSO, J., GREEN, D. and SÁNCHEZ ALVARADO, A. (2010). Cell death and tissue remodeling in planarian regeneration Dev Biol 338: 76-85.

PELLETTIERI, J. and SÁNCHEZ ALVARADO, A. (2007). Cell Turnover and Adult Tissue Homeostasis: From Humans to Planarians. Annu Rev Genet 41: 83-105.

REDDIEN, P., BERMANGE, A., MURFITT, K., JENNINGS, J. and SÁNCHEZ ALVARADO, A. (2005a). Identification of genes needed for regeneration, stem cell function, and tissue homeostasis by systematic gene perturbation in planaria. Dev Cell 8: 635-649.

REDDIEN, P., OVIEDO, N., JENNINGS, J., JENKIN, J. and SÁNCHEZ ALVARADO, A. (2005b). SMEDWI-2 is a PIWI-like protein that regulates planarian stem cells. Science 310: 1327-1330.

ROBB, S., ROSS, E. and SÁNCHEZALVARADO, A. (2007). SmedGD: the Schmidtea mediterranea genome database. Nucleic Acids Res 36: D599-D606.

ROMERO, R. and BAGUÑÁ, J. (1991). Quantitative cellular analysis of growth and reproduction in freshwater planarians (Turbellaria; Tricladida). I. A cellular description of the intact organism. Invertebr Reprod Dev 19: 157-165.

ROUHANA, L., SHIBATA, N., NISHIMURA, O. and AGATA, K. (2010). Different requirements for conserved post-transcriptional regulators in planarian regeneration and stem cell maintenance. Dev Biol 341: 429-43.

SALVETTI, A., ROSSI, L., LENA, A., BATISTONI, R., DERI, P., RAINALDI, G., LOCCI, M., EVANGELISTA, M. and GREMIGNI, V. (2005). DjPum, a homologue of Drosophila Pumilio, is essential to planarian stem cell maintenance. Development 132: 1863-1874.

SAMANI, A., YAKAR, S., LEROITH, D. and BRODT, P. (2007). The role of the IGF system in cancer growth and metastasis: overview and recent insights. Endocr Rev 28: 20-47.

SAMBROOK, J. and RUSSELL, D. (2001). Molecular Cloning. Molecular cloning: a laboratory manual. Irwin N, Janssen K. A, editors. Cold Spring Harbor: Cold Spring Harbor Laboratory Press.

SCHULTZ, J., MILPETZ, F., BORK, P. and PONTING, C. (1998). SMART, a simple modular architecture research tool: Identification of signaling domains Proc. Natl. Acad. Sci. USA 95: 5857-5864.

SMIT, A., VANKESTEREN, R., LI, K., VAN MINNEN, J., SPIJKER, S., VANHEERIKHUIZEN, H. and GERAERTS, W. (1998). Towards understanding the role of insulin in the brain: lessons from insulin-related signaling systems in the invertebrate brain. Prog Neurobiol 54: 35-54.

TATAR, M., BARTKE, A. and ANTEBI, A. (2003). The endocrine regulation of aging by insulin-like signals. Science 299: 1346-1351.

TIMMONS, L., COURT, D. and FIRE, A. (2001). Ingestion of bacterially expressed dsRNAs can produce specific and potent genetic interference in Caenorhabditis elegans. Gene 263: 103-112.

UEISHI, S., SHIMIZU, H. and INOUE, Y. (2009). Male germline stem cell division and spermatocyte growth require insulin signaling in Drosophila. Cell Struct Funct 34: 61-69.

WAGNER, D., WANG, I. and REDDIEN, P. (2011). Clonogenic neoblasts are pluripotent adult stem cells that underlie planarian regeneration. Science 332: 811-816.

WANG, Y., STARY, J., WILHELM, J. and NEWMARK, P. (2010). A functional genomic screen in planarians identifies novel regulators of germ cell development. Genes Dev 24: 2081-2092.

WANG, Y., ZAYAS, R., GUO, T. and NEWMARK, P. (2007). nanos function is essentia for development and regeneration of planarian germ cells. Proc. Natl. Acad. Sci. USA 104: 5901-5906.

WENEMOSER, D. and REDDIEN, P. (2010). Planarian regeneration involves distinct stem cell responses to wounds and tissue absence. Dev Biol 344: 979-991.

WU, Q. and BROWN, M. (2006). Signaling and function of insulin-like peptides in insects. Annu Rev Entomol 51: 1-24

ZAYAS, R., HERNANDEZ, A., HABERMANN, B., WANG, Y., STARY, J. and NEWMARK, P. (2005). The planarian Schimdtea mediterranea as a model for epigenetic germ cell specification: Analysis of ESTs from the hermaphroditic strain. Proc. Natl. Acad. Sci. USA 102: 18491-18496. 


\section{Further Related Reading, published previously in the Int. J. Dev. Biol.}

Diverse miRNA spatial expression patterns suggest important roles in homeostasis and regeneration in planarians Cristina González-Estévez, Varvara Arseni, Roshana S. Thambyrajah, Daniel A. Felix and A. Aziz Aboobaker Int. J. Dev. Biol. (2009) 53: 493-505

Characterization of novel genes expressed specifically in the sexual organs of the planarian Dugesia ryukyuensis Sumitaka Hase, Emiko Kashiwagi, Kazuya Kobayashi, Motonori Hoshi and Midori Matsumoto Int. J. Dev. Biol. (2007) 51: 345-349

Expression of DjXnp, a novel member of the SNF2-like ATP-dependent chromatin remodelling genes, in intact and regenerating planarians.

Leonardo Rossi, Paolo Deri, Ilaria Andreoli, Vittorio Gremigni, Alessandra Salvetti and Renata Batistoni

Int. J. Dev. Biol. (2003) 47: 293-298

Planarian pharynx regeneration revealed by the expression of myosin heavy chain-A.

Tomoko Sakai, Kentaro Kato, Kenji Watanabe and Hidefumi Orii

Int. J. Dev. Biol. (2002) 46: 329-332

Effects of activators and antagonists of the neuropeptides substance P and substance $\mathrm{K}$ on cell proliferation in planarians. J Baguñà, E Saló and R Romero Int. J. Dev. Biol. (1989) 33: 261-266

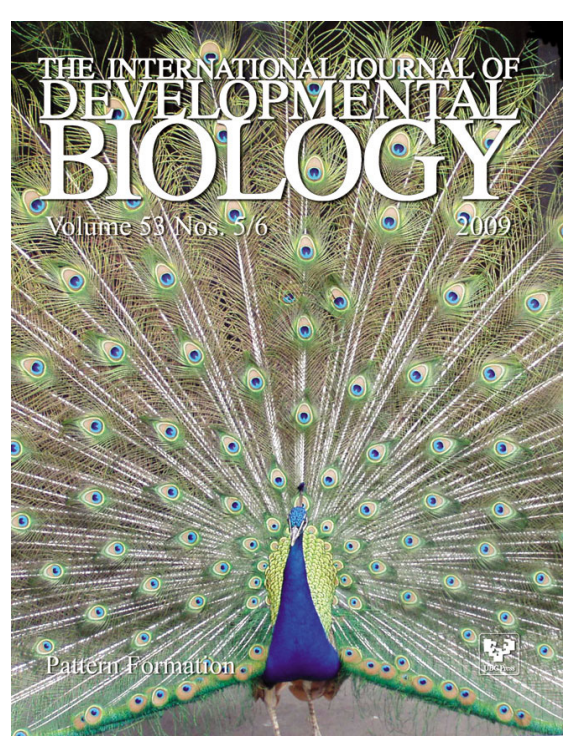
5 yr ISI Impact Factor $(2010)=2.961$
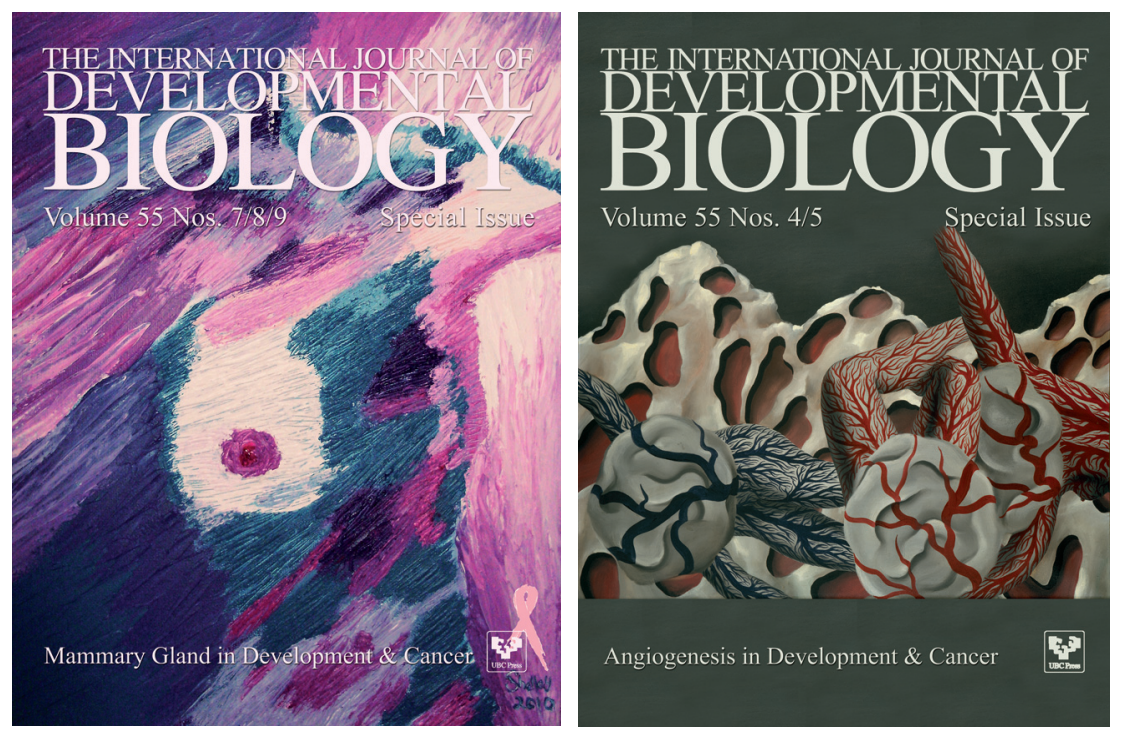